\title{
ON COMBINATORIAL CRITERIA FOR NON-DEGENERATE SINGULARITIES
}

\author{
Szymon Brzostowski AND GrZEGORZ OLEKSIK
}

\begin{abstract}
In this article we give a sufficient and necessary condition for a Kouchnirenko nondegenerate holomorphic function to have an isolated singularity at 0 in terms of its support. As a corollary we give some useful sufficient conditions for singularity to be isolated.
\end{abstract}

\section{Introduction}

Let $f:\left(\mathbf{C}^{n}, 0\right) \rightarrow(\mathbf{C}, 0)$ be the germ of a holomorphic function. One of the problems in the theory of singularities is to check effectively that $f$ has an isolated singularity at 0 . Many authors deal with this problem in various context. For instance, by the local Nullstellensatz, $f$ has an isolated singularity at 0 if and only if the Milnor number $\mu(f)$ is finite. Similarly, the Lojasiewicz exponent $£_{0}(f)$ is finite if and only if $f$ has an isolated singularity at 0 (for definitions see Preliminaries).

Kouchnirenko in [9] gave for a set $M \subset \mathbf{N}^{n}$ a necessary and sufficient condition that $f$ with supp $f \subset M$, has an isolated singularity at 0 (see Theorem 2.8). Other authors: Wall [22], Orlik and Randell [16], Shcherbak [21] obtained similar results. One can find more historical comments on this topic in [15] and [7].

The quasihomogeneous case was considered by the authors mentioned above as well as by Saito ([19], [20]), Kreuzer and Skarke [10], Hertling and Kurbel [7].

In this paper we examine the problem in the class of non-degenerate holomorphic functions. As the main result we prove that a non-degenerate function (see Preliminaries for the definition) with the support satisfying a combinatorial condition has an isolated singularity at 0 (Theorem 3.1). As a corollary we give

Mathematics Subject Classification. 32S05.

Key words and phrases. Isolated singularity, non-degeneracy in the Kouchnirenko sense, Milnor number, Newton number.

The paper was partially supported by the Polish National Science Centre (NCN), Grant No 2012/ 07/B/ST1/03293.

Received July 29, 2014; revised September 18, 2015. 
some useful sufficient conditions for a holomorphic function to have an isolated singularity at 0 (Corollary 3.16). We also prove that Kouchnirenko condition for $M$ is equivalent to the finiteness of the Newton number of $\lambda_{M}$ (Corollary 3.12). It was announced already by Kouchnirenko [8, Remarque 1.13 (ii)] but without a proof. C. T. C. Wall considered different type of non-degeneracy from the Kouchnirenko one. He got similar results for his non-degeneracy to the ones obtained in this paper (see Lemma 1.2 and Theorem 1.6 in [23]).

We also explain some details concerning non-convenient singularities. Kouchnirenko in his celebrated paper gave the formula for the Milnor number only in the convenient case [8, Théorème I (ii)]. Consequently, many authors cited this formula only in this case. For example Damon and Gaffney wrote "Note that Kouchnirenko only carries out his analysis for fit germs" [4, Section 2] and Wall wrote "Although Kouchnirenko gives rather general definition of non-degeneracy, his main results are proved only for function satisfying an additional condition called (in French) "commode" [23]. However, Kouchnirenko did prove his formula also for non-convenient functions (see [8, Section 3]). Therefore, we explicitly give the formula for the Milnor number (Corollary 3.10) without the assumption that function is convenient. Kouchnirenko proved this formula for non-convenient functions using [8, Théorème 3.7]. We will use Lemma 3.8 instead.

In Appendix we give the effective bound for the constant $C$ of Lemma 3.6. This bound is expressed in terms of the Lojasiewicz exponent. This invariant may be effectively computed using e.g. Gröbner basis techniques (see [18]) or may be estimated (see [5]).

\section{Preliminaries}

Let $f:\left(\mathbf{C}^{n}, 0\right) \rightarrow(\mathbf{C}, 0)$ be a non-zero holomorphic function in an open neighborhood of $0 \in \mathbf{C}^{n}$. We say that $f$ has a singularity at 0 if $f(0)=0$, $\nabla f(0)=0$, where $\nabla f=\left(f_{z_{1}}, \ldots, f_{z_{n}}\right)$. It is equivalent to the condition ord $f \geq 2$, where ord $f$ means the order of $f$ at 0 . We say that $f$ has an isolated singularity at 0 if $f$ has an isolated critical point at the origin i.e., additionally $\nabla f(z) \neq 0$ for $z \neq 0$ near 0 . We denote $\mathbf{N}=\{0,1,2, \ldots\}$ and $\mathbf{R}_{+}=\{x \in \mathbf{R}$ : $x \geq 0\}$. Let $\sum_{v \in \mathbf{N}^{n}} a_{v} z^{v}$ be the Taylor expansion of $f$ at 0 . We define the set supp $f$ by supp $f=\left\{v \in \mathbf{N}^{n}: a_{v} \neq 0\right\}$ and call it the support of $f$. Let $w_{1}, \ldots, w_{n}, d$ be positive rational numbers. A polynomial $f \in \mathbf{C}\left[z_{1}, \ldots, z_{n}\right]$ is called quasihomogeneous of type $\left(w_{1}, \ldots, w_{n} ; d\right)$ if

$$
\sum_{i=1}^{n} v_{i} w_{i}=d \quad \text { for any } v \in \operatorname{supp} f .
$$

The numbers $w_{1}, \ldots, w_{n}$ are called weights of $f$ and the number $d$ is called weighted degree of $f$. We define

$$
\Gamma_{+}(f)=\operatorname{conv}\left\{v+\mathbf{R}_{+}^{n}: v \in \operatorname{supp} f\right\} \subset \mathbf{R}^{n}
$$


and call it the Newton diagram of $f$. Let $u \in \mathbf{R}_{+}^{n} \backslash\{0\}$. Put

$$
\begin{aligned}
l\left(u, \Gamma_{+}(f)\right) & =\inf \left\{\langle u, v\rangle: v \in \Gamma_{+}(f)\right\}, \\
\Delta\left(u, \Gamma_{+}(f)\right) & =\left\{v \in \Gamma_{+}(f):\langle u, v\rangle=l\left(u, \Gamma_{+}(f)\right)\right\} .
\end{aligned}
$$

We say that $S \subset \mathbf{R}^{n}$ is a face of $\Gamma_{+}(f)$ if $S=\Delta\left(u, \Gamma_{+}(f)\right)$ for some $u \in \mathbf{R}_{+}^{n} \backslash\{0\}$. The vector $u$ is called a vector supporting $S$. It is easy to see that $S$ is a closed and convex set and $S \subset \operatorname{Fr}\left(\Gamma_{+}(f)\right)$, where $\operatorname{Fr}(A)$ denotes the boundary of $A$. One can check that a face $S \subset \Gamma_{+}(f)$ is compact if and only if there exists a vector supporting $S$ which has all coordinates positive. We call the family of all compact faces of $\Gamma_{+}(f)$ the Newton boundary of $f$ and denote it by $\Gamma(f)$. We denote by $\Gamma^{k}(f)$ the set of all compact $k$-dimensional faces of $\Gamma_{+}(f), k=0, \ldots$, $n-1$. For each compact face $S \in \Gamma(f)$ we define quasihomogeneous polynomial $f_{S}=\sum_{v \in S} a_{v} z^{v}$. We say that $f$ is non-degenerate on the face $S \in \Gamma(f)$ if the system of equations

$$
\frac{\partial f_{S}}{\partial z_{1}}=\cdots=\frac{\partial f_{S}}{\partial z_{n}}=0
$$

has no solution in $\left(\mathbf{C}^{*}\right)^{n}$, where $\mathbf{C}^{*}=\mathbf{C} \backslash\{0\}$. We say that $f$ is non-degenerate in the sense of Kouchnirenko (shortly non-degenerate) if it is non-degenerate on each face of $\Gamma(f)$. We say that $f$ is convenient if $\Gamma_{+}(f)$ has non-empty intersection with each coordinate axis. We say that $f$ is nearly convenient if the distance of $\Gamma_{+}(f)$ to each coordinate axis does not exceed 1. Denote by $\mathcal{O}_{n}$ the local ring of germs of holomorphic functions in $n$-variables at $0 \in \mathbf{C}^{n}$. Let us recall that the Milnor Number $\mu(f)$ is defined as $\mu(f)=\operatorname{dim}_{\mathbf{C}} \mathcal{O}^{n} /\left(f_{z_{1}}^{\prime}, \ldots, f_{z_{n}}^{\prime}\right)$. Moreover, the Newton number $v(f)$ for convenient $f$ is defined as

$$
v(f)=n ! V_{n}-(n-1) ! V_{n-1}+\cdots+(-1)^{n} V_{0},
$$

where $V_{i}$ denotes the sum of $i$-dimensional volumes of the intersection of the cone (with apex at 0 ) spanned by $\Gamma(f)$ with the coordinate subspaces of dimension $i$. We may also define the Newton number for non-convenient holomorphic function (see [8, Définition 1.9]). Namely, let $f$ be non-convenient and $I \subset\{1,2, \ldots, n\}$ be a non-empty set such that $\Gamma_{+}(f) \cap O X_{i}=\emptyset$ for $i \in I$ and $\Gamma_{+}(f) \cap O X_{i} \neq \emptyset$ for $i \notin I$. We define

$$
v(f)=\sup _{m \in \mathbf{N}} v\left(f+\sum_{i \in I} z_{i}^{m}\right) .
$$

Now, we recall some known results concerning support of holomorphic function having an isolated singularity at 0 . Kouchnirenko in [9, Theorem 1] gave for a set $M \subset \mathbf{N}^{n}$ a necessary and sufficient condition for existence of $f$, supp $f \subset M$, having an isolated singularity at 0 . In addition, one can deduce from his reasoning that if $M$ satisfies this condition, every holomorphic function $f$, supp $f \subset M$, ord $f \geq 2$, with generic coefficients has an isolated singularity at 0 . Before giving his result we state definitions. 
Let $M \subset \mathbf{N}^{n}$. Define the sets $M_{i}=\left\{v \in \mathbf{N}^{n}: v+e_{i} \in M\right\}$, where $e_{1}, \ldots, e_{n}$, is the standard basis in $\mathbf{R}^{n}$. Notice that if we take $\lambda_{M}(z)=\sum_{m \in M} z^{m}$ then $M_{i}=\operatorname{supp} \partial \lambda_{M} / \partial z_{i}$ for every $i=1,2, \ldots, n$.

Let $I \subset\{1, \ldots, n\}$ be a non-empty set. Set

$$
O X_{I}=\left\{x \in \mathbf{R}^{n}: x_{i}=0 \text { for } i \notin I\right\} .
$$

We may notice that $O X_{I}$ is the hyperplane spanned by the axes $O X_{i}, i \in I$. We say that $M$ satisfies the Kouchnirenko condition for $I$ if there exist at least $|I|$ nonempty sets among the sets $M_{1} \cap O X_{I}, M_{2} \cap O X_{I}, \ldots, M_{n} \cap O X_{I}$. We say that $M$ satisfies the Kouchnirenko condition if $M$ satisfies the Kouchnirenko condition for every $I \subset\{1,2, \ldots, n\}$.

Remark 2.1. If $M$ satisfies the Kouchnirenko condition, it may happen that $\lambda_{M}$ does not have an isolated singularity at 0 . For example let $\lambda_{M}(z)=$ $\left(z_{1}+z_{2}\right)\left(z_{1}+z_{3}\right)$. It is easy to check that $\lambda_{M}$ does not have an isolated singularity at 0 and that is degenerate on the face $S=\operatorname{conv}\left\{\operatorname{supp}\left(\lambda_{M}\right)\right\}$.

Example 2.2. a) Let $f\left(z_{1}, z_{2}\right)=z_{1}^{2}+z_{1} z_{2}$. We shall show that $\operatorname{supp} f$ satisfies the Kouchnirenko condition. Put $M=\operatorname{supp} f$. Then $M_{1}=\{(0,1)$, $(1,0)\}, \quad M_{2}=\{(1,0)\}$. If $I=\{1,2\}$ we easily check that $M$ satisfies the Kouchnirenko condition for $I$. If $I=\{1\}$ or $I=\{2\}$, then $M_{1} \cap O X_{1} \neq \emptyset$.

b) Let $f\left(z_{1}, z_{2}, z_{3}\right)=z_{1}\left(z_{1}+z_{2}+z_{3}\right)$. We shall show that supp $f$ does not satisfy the Kouchnirenko condition. Indeed, take $I=\{2,3\}$ then $|I|=2$ but $M_{1} \cap O X_{I} \neq \emptyset$ and $M_{2} \cap O X_{I}=M_{3} \cap O X_{I}=\emptyset$.

Now, we explain the Kouchnirenko condition for $I$ in the extreme cases $|I|=1$ and $|I|=n$. It is easy to check the following property.

Property 2.3. Let $f:\left(\mathbf{C}^{n}, 0\right) \rightarrow(\mathbf{C}, 0)$ be a holomorphic function which has a singularity at 0 . The following holds:

(i) supp $f$ satisfies the Kouchnirenko condition for every $I=\{i\}, \quad i=1$, $2, \ldots, n$ if and only if $f$ is nearly convenient,

(ii) supp $f$ satisfies the Kouchnirenko condition for $I=\{1,2, \ldots, n\}$ if and only if $f_{z_{i}}^{\prime} \neq 0, i=1,2, \ldots, n$.

The next simple property shows that the Kouchnirenko condition for supp $f$ implies that the Newton diagram of holomorphic function $f$ which defines an isolated singularity at 0 , has non-empty intersection with every $(n-1)$ dimensional coordinate hyperplane in $\mathbf{R}^{n}, n \geq 3$.

Property 2.4. Let $f:\left(\mathbf{C}^{n}, 0\right) \rightarrow(\mathbf{C}, 0), n \geq 3$, be a holomorphic function which has a singularity at 0 . If supp $f$ satisfies the Kouchnirenko condition then $\Gamma_{+}(f) \cap O X_{I} \neq \emptyset$ for every set $I \subset\{1,2, \ldots, n\},|I|=n-1$. 
The following two propositions, which are easy consequences of the definition, give conditions equivalent to the Kouchnirenko condition for supp $f$ in terms of the Newton diagram of $f$ in two and three variables.

Proposition 2.5. Let $f:\left(\mathbf{C}^{2}, 0\right) \rightarrow(\mathbf{C}, 0)$ be a holomorphic function which has a singularity at 0 . Then the following conditions are equivalent:

(i) $f$ is nearly convenient,

(ii) supp $f$ satisfies the Kouchnirenko condition.

Proposition 2.6. Let $f:\left(\mathbf{C}^{3}, 0\right) \rightarrow(\mathbf{C}, 0)$ be a holomorphic function which has a singularity at 0 . Then the following conditions are equivalent:

(i) $f$ is nearly convenient and $\Gamma_{+}(f) \cap O X_{\{i, j\}} \neq \emptyset$ for every $i, j \in\{1,2,3\}$, $i \neq j$

(ii) supp $f$ satisfies the Kouchnirenko condition.

There are some combinatorial conditions equivalent to the Kouchnirenko condition. Hertling and Kurbel collected such conditions for a quasihomogeneous polynomial in [7, Lemma 2.1] but their lemma is also true without the assumption of quasihomogeneity.

Lemma 2.7. Let $M \subset \mathbf{N}^{n}$ and ord $\lambda_{M} \geq 2$. Set $S_{I}=\left\{k: M_{k} \cap O X_{I} \neq \emptyset\right\}$, $I \subset\{1,2, \ldots, n\}$. Then the following conditions are equivalent:

(K) $\# I \leq \# S_{I}$ for $\forall I$ (the Kouchnirenko condition for $M$ )

$\left(\mathrm{K}^{\prime}\right) \quad \# I \leq \# S_{I}$ for $\forall I$ with $1 \leq \# I \leq \frac{n+1}{2}$

(C1) $\left[M \cap O X_{I}=\emptyset \Rightarrow \# I \leq \#\left(S_{I} \backslash I\right)\right]$ for $\forall I$

$\left(\mathrm{C} 1^{\prime}\right)\left[M \cap O X_{I}=\emptyset \Rightarrow \# I \leq \#\left(S_{I} \backslash I\right)\right]$ for $\forall I$ with $1 \leq \# I \leq \frac{n+1}{2}$

(C2) If $\# J<\# I$, then $S_{I} \backslash J \neq \emptyset$.

Proof. The proof is the same as the proof of Lemma 2.1 in [7].

Now, we recall Theorem 1 in [9].

THEOREM 2.8 ([9, Theorem 1]). Let $M \subset \mathbf{N}^{n}$ and ord $\lambda_{M} \geq 2$. Then the following conditions are equivalent:

(ISe) there exists an isolated singularity $f:\left(\mathbf{C}^{n}, 0\right) \rightarrow(\mathbf{C}, 0)$ such that supp $f \subset M$,

(K) M satisfies the Kouchnirenko condition.

As a direct consequence of Theorem 2.8 we get the following corollary.

COROLlary 2.9. If $f$ has an isolated singularity at 0 , then the support of $f$ satisfies the Kouchnirenko condition. 
Remark 2.10. It seems that Saito [19, Lemma 1.5] was the first to state the corollary above, since he proved that a support of holomorphic function having an isolated singularity at 0 , satisfies condition $(\mathrm{C} 1)$, which by Lemma 2.7 is equivalent to the Kouchnirenko condition. It can also be extracted from [21, Remark 3].

As a direct consequence of the corollary above and Property 2.3(i) we give the following property.

PROPERTY 2.11. If $f$ has an isolated singularity at 0 , then $f$ is nearly convenient.

\section{Main result}

We begin with the main result.

THEOREM 3.1. Let $f:\left(\mathbf{C}^{n}, 0\right) \rightarrow(\mathbf{C}, 0), n \geq 2$ be a non-degenerate holomorphic function which has a singularity at 0 . If supp $f$ satisfies the Kouchnirenko condition, then $f$ has an isolated singularity at 0 .

We deduce the main theorem follows from the one below.

THEOREM 3.2. Let $f:\left(\mathbf{C}^{n}, 0\right) \rightarrow(\mathbf{C}, 0), n \geq 2$ be a non-degenerate holomorphic function which has a singularity at 0 . If $v(f)$ is finite, then $f$ has an isolated singularity at 0 .

In fact, in Corollary 3.12 below we will show that Kouchnirenko condition for supp $f$ is equivalent to the finiteness of $v(f)$. This together with Theorem 3.2 gives Theorem 3.1.

Remark 3.3. Theorem 3.1 was given by Lenarcik [11, Property 3.2] in the case $n=2$ and by the second author in [15, Theorem 5.4] in the case $n \leq 3$. It also confirms Conjecture 5.5 stated in [15].

Remark 3.4. By Theorem 3.1 and Corollary 2.9 we see that in the class of non-degenerate function having a singularity at 0 the Kouchnirenko condition for supp $f$ is equivalent that $f$ defines an isolated singularity at 0 .

Now, we give some lemmas and propositions needed in the proof of Theorem 3.2. The following proposition was discovered independently by many authors, see for example [8, Théorème I (ii)], [13, Remark 2.7], [3, Proposition 4.4], [15, Corollary 5.8].

Proposition 3.5. If $f$ is convenient and non-degenerate, then $f$ has an isolated singularity at 0 . 
The lemma below says that the Newton number of a non-convenient holomorphic function is independent of the way we make it convenient. More precisely, we have the following lemma.

Lemma 3.6. Let $f:\left(\mathbf{C}^{n}, 0\right) \rightarrow(\mathbf{C}, 0), n \geq 2$ be a holomorphic function. Assume that $v(f)$ is finite. Let $I=\left\{i_{1}, \ldots, i_{k}\right\} \subset\{1,2, \ldots, n\}$ be a non-empty set such that $\Gamma_{+}(f) \cap O X_{i}=\emptyset$ for $i \in I$ and $\Gamma_{+}(f) \cap O X_{i} \neq \emptyset$ for $i \notin I$. Then there exists $C \geq 2$ such that

$$
v\left(f+\sum_{i \in I} z_{i}^{m_{i}}\right)=v(f) \quad \text { for every } m_{i} \geq C, i \in I
$$

Proof. Without loss of generality we may assume that $I=\{1,2, \ldots, k\}$. Put $f_{m}=f+\sum_{i=1}^{k} z_{i}^{m}, \quad m \geq 2$. By assumption $v(f)=\sup _{m \in \mathbf{N}} v\left(f_{m}\right)<\infty$. Since $\Gamma_{+}\left(f_{m+1}\right) \subset \Gamma_{+}\left(f_{m}\right)$, by monotonicity of the Newton number (see for example [6]) we have $v\left(f_{m}\right) \leq v\left(f_{m+1}\right)$. Therefore the sequence $v\left(f_{m}\right)$ is convergent. Since $v\left(f_{m}\right) \in \mathbf{N}$, we get that there exists $C$ such that

$$
v\left(f_{m}\right)=v(f) \text { for } m \geq C .
$$

Take $m_{1}, \ldots, m_{k} \geq C$. Set $m_{\max }:=\max \left\{m_{1}, \ldots, m_{k}\right\}, m_{\min }:=\min \left\{m_{1}, \ldots, m_{k}\right\}$. From the inclusion

$$
\Gamma_{+}\left(f+\sum_{i=1}^{k} z_{i}^{m_{\max }}\right) \subset \Gamma_{+}\left(f+\sum_{i=1}^{k} z_{i}^{m_{i}}\right) \subset \Gamma_{+}\left(f+\sum_{i=1}^{k} z_{i}^{m_{\min }}\right),
$$

and monotonicity of the Newton number and (1) we infer

$$
v\left(f+\sum_{i=1}^{k} z_{i}^{m_{i}}\right)=v(f) .
$$

The next lemma allows us to make $f$ both convenient and non-degenerate.

Lemma 3.7. Let $f:\left(\mathbf{C}^{n}, 0\right) \rightarrow(\mathbf{C}, 0), n \geq 2$, be a non-degenerate holomorphic function which has a singularity at 0 . Assume that $\Gamma_{+}(f) \cap O X_{i}=\emptyset$ for some $i \in\{1,2, \ldots, n\}$. Then there exists $C \geq 2$ such that $f_{i}=f+z_{i}^{m}$ is non-degenerate for every $m \geq C$.

Proof. Let $S \in \Gamma(f)$. Since $S$ is compact we can choose $u_{S} \in(0, \infty)^{n}$ such that $S=\Delta\left(u_{S}, \Gamma_{+}(f)\right)$ (see Preliminaries). Put

$$
W=\bigcup_{S \in \Gamma(f)}\left\{v \in \mathbf{R}_{+}^{n}:\left\langle u_{S}, v\right\rangle \leq l\left(u_{S}, \Gamma_{+}(f)\right)\right\} .
$$

It is easy to check that $W$ is compact and intersects every coordinate axis. Hence we may choose $C \geq 2$ so large that the points in $\mathbf{R}_{+}^{n}$ determined by the monomials $z_{i}^{m}, m \geq C$, do not lie in $W$. Let $m \geq C$. We show that 
$f_{i}=f+z_{i}^{m}$ is a non-degenerate. Let $P \in \mathbf{R}^{n}$ be the point determined by the monomial $z_{i}^{m}$. From the choice of $C$, we observe that $P$ is a vertex of $\Gamma\left(f_{i}\right)$. By nearly-convenience of $\Gamma_{+}(f)$ there exists a point $Q$, which is at distance 1 from the axis $O X_{i}$. Hence the segment $\overline{P Q}$ is a face of $\Gamma\left(f_{i}\right)$ and $\Gamma^{1}\left(f_{i}\right) \neq \emptyset$. Therefore we get $\Gamma\left(f_{i}\right)=\Gamma(f) \cup \Delta$, where $\Delta$ is the family of the faces in $\Gamma\left(f_{i}\right)$ containing $P$ as a vertex. Since $f$ is non-degenerate, $f_{i}$ is non-degenerate on each face of $\Gamma(f)$. Now take $\delta \in \Delta \cap \Gamma^{k}\left(f_{i}\right), k \geq 1$. From the choice of $C$ we get that $\delta$ is the convex hull of $P$ and some face $\sigma \in \Gamma(f)$ such that $\operatorname{dim} \sigma<\operatorname{dim} \delta$. Therefore $\left(f_{i}\right)_{\delta}=z_{i}^{m}+f_{\sigma}$, where $f_{\sigma}$ is a quasihomogeneous polynomial. Since $\operatorname{dim} \sigma<n-1$, the weights of $f_{\sigma}$ are not uniquely determined. Let $\left(f_{i}\right)_{\delta}$ be of type $\left(w_{1}, \ldots, w_{i-1}, 1 / m, w_{i+1}, \ldots, w_{n} ; 1\right)$. Take $m^{\prime}>m$ and consider the polynomial $f_{\sigma}+z_{i}^{m^{\prime}}$. Let $P^{\prime} \in \mathbf{R}_{+}^{n}$ be the point determined by the monomial $z_{i}^{m^{\prime}}$. Since $\sigma$ is compact and $\sigma \in \Gamma(f)$, the set $\operatorname{conv}\left(\sigma, P^{\prime}\right)$ is also compact and there exist positive weights $\left(w_{1}^{\prime}, \ldots, w_{i-1}^{\prime}, 1 / m^{\prime}, w_{i+1}^{\prime}, \ldots, w_{n}^{\prime} ; 1\right)$ of $f_{\sigma}+\left(z_{i}\right)^{m^{\prime}}$. Thus $f_{\sigma}$ is simultaneously of the types

$$
\left(w_{1}, \ldots, w_{i-1}, 1 / m, w_{i+1}, \ldots, w_{n} ; 1\right) \text { and }\left(w_{1}^{\prime}, \ldots, w_{i-1}^{\prime}, 1 / m^{\prime}, w_{i+1}^{\prime}, \ldots, w_{n}^{\prime} ; 1\right) .
$$

Using Euler's formula for these weights we get

$$
\sum_{j \neq i}\left(w_{j}-w_{j}^{\prime}\right) z_{j} \frac{\partial f_{\sigma}}{\partial z_{j}}+\left(\frac{1}{m}-\frac{1}{m^{\prime}}\right) z_{i} \frac{\partial f_{\sigma}}{\partial z_{i}}=0
$$

Now we show that $f_{i}$ is non-degenerate on $\delta$. Suppose to the contrary that there exists $z^{0} \in\left(\mathbf{C}^{*}\right)^{n}$, such that $\nabla\left(f_{i}\right)_{\delta}\left(z^{0}\right)=0$. Hence $\left(f_{\sigma}\right)_{z_{i}}\left(z^{0}\right)=0$ for $j \neq i$. By (2) we get also $z_{i}^{0}\left(f_{\sigma}\right)_{z_{i}}^{\prime}\left(z^{0}\right)=0$. Summing up, $\nabla f_{\sigma}\left(z^{0}\right)=0$, which contradicts non-degeneracy of $f$ on the face $\sigma$.

Now, using induction we extend the previous lemma as follows.

LEMma 3.8. Let $f:\left(\mathbf{C}^{n}, 0\right) \rightarrow(\mathbf{C}, 0), n \geq 2$, be a non-degenerate holomorphic function. Let $I=\left\{i_{1}, \ldots, i_{k}\right\} \subset\{1,2, \ldots, n\}$ be a non-empty subset such that $\Gamma_{+}(f) \cap O X_{i}=\emptyset$ for $i \in I$ and $\Gamma_{+}(f) \cap O X_{i} \neq \emptyset$ for $i \notin I$. Then for every $C \geq 2$ there exist $m_{1}, \ldots, m_{k} \geq C$ such that $f_{k}=f+\sum_{j=1}^{k} z_{i_{j}}^{m_{j}}$ is non-degenerate and convenient.

Proof. Without loss of generality we may assume that $I=\{1,2, \ldots, k\}$. For every $j=0,1, \ldots, k$ denote by $\left(A_{j}\right)$ the assertion "For every $C \geq 2$ there exist $m_{1}, \ldots, m_{j} \geq C$ such that $f_{j}=f+\sum_{i=1}^{j} z_{i}^{m_{i}}$ is non-degenerate." We show inductively (with respect to $j$ ) that $\left(A_{j}\right)$ holds for every $j=0,1, \ldots, k$. The assertion is true for $j=0$ by the assumption. Let $j \in\{0,1, \ldots, k\}$. Suppose that $\left(A_{j}\right)$ is true. We show that $\left(A_{j+1}\right)$ is also true. Let $C \geq 2$. Since $\left(A_{j}\right)$ is true there exist $m_{1}, \ldots, m_{j} \geq C$ such that $f_{j}=f+\sum_{i=1}^{j} z_{i}^{m_{i}}$ is non-degenerate. By Lemma 3.7 there exists $m_{j+1} \geq C$ such that $f_{j+1}=f_{j}+z_{j+1}^{m_{j+1}}$ is non-degenerate. By induction $\left(A_{j}\right)$ is true for every $j=0,1, \ldots, k$. In particular, $\left(A_{k}\right)$ is true. 
Remark 3.9. We may notice that this way we are able to prove a stronger version of the lemma above, namely it is also true for $f_{k}=f+\sum_{j=1}^{k} \alpha_{j} z_{i_{j}}^{m_{j}}$ with arbitrary $\alpha_{j} \neq 0$ (compare [8, Théorème 3.7]).

Proof of Theorem 3.2. If $f$ is convenient, the assertion follows from Proposition 3.5. Suppose that $f$ is not convenient. Let $I=\left\{i_{1}, \ldots, i_{k}\right\} \subset$ $\{1,2, \ldots, n\}$ be a set such that $\Gamma_{+}(f) \cap O X_{i}=\emptyset$ for $i \in I$ and $\Gamma_{+}(f) \cap O X_{i} \neq$ $\emptyset$ for $i \notin I$. Without loss of generality we may assume that $I=\{1,2, \ldots, k\}$. By Lemma 3.6 there exists $C \geq 2$ such that

$$
v\left(f+\sum_{i=1}^{k} z_{i}^{m_{i}}\right)=v(f) \text { for every } m_{i} \geq C, i=1, \ldots, k .
$$

By Lemma 3.8 there exist $m_{1}, \ldots, m_{k} \geq \max \{C, v(f)+1\}$ such that $f_{k}=$ $f+\sum_{i=1}^{k} z_{i}^{m_{i}}$ is non-degenerate and convenient. By Proposition 3.5 we get that $f_{k}$ has an isolated singularity at 0 . Hence by (3) and Théorème I (ii) in [8], we get

$$
\operatorname{ord}\left(f_{k}-f\right)=\min _{i=1}^{k} m_{i} \geq v(f)+1=v\left(f_{k}\right)+1=\mu\left(f_{k}\right)+1 .
$$

From (4) and since $f_{k}$ is $\mu\left(f_{k}\right)+1$ right determined (see for example [2, Section 6.3]) we get that $f$ and $f_{k}$ are right (biholomorphically) equivalent. This implies $f$ has an isolated singularity at 0 and

$$
\mu(f)=\mu\left(f_{k}\right) .
$$

From the proof above we easily get Théorème I (ii) in [8] without the assumption that $f$ is convenient. More precisely, we get the following corollary.

Corollary 3.10. Let $f:\left(\mathbf{C}^{n}, 0\right) \rightarrow(\mathbf{C}, 0), n \geq 1$, be a non-degenerate holomorphic function which defines an isolated singularity at 0 . Then $\mu(f)=v(f)$.

Proof. If $f$ is a convenient singularity, the assertion follows from Théorème I (ii) in [8]. In the opposite case, repeating the proof of Theorem 3.1 with the same notations and by (5), Théorème I (ii) in [8] and (3) we get

$$
\mu(f)=\mu\left(\hat{f}_{k}\right)=v\left(\hat{f}_{k}\right)=v(f) .
$$

As a direct consequence of Theorem 3.2 and Corollary 3.10, we get the following.

COROllary 3.11. Let $f:\left(\mathbf{C}^{n}, 0\right) \rightarrow(\mathbf{C}, 0), n \geq 1$, be a non-degenerate holomorphic function which has a singularity at 0 . Then $\mu(f)<\infty \Leftrightarrow v(f)<\infty$.

The next corollary says that Kouchnirenko condition for $M$ is equivalent to the finiteness of the Newton number of $\lambda_{M}$. It was announced already by Kouchnirenko [8, Remarque 1.13 (ii)] but without proof. 
Corollary 3.12. Let $M \subset \mathbf{N}^{n}$ and ord $\lambda_{M} \geq 2$. A set $M$ satisfies the Kouchnirenko condition if and only if $v\left(\lambda_{M}\right)$ is finite.

Proof. " $\Rightarrow$ " Suppose that $M$ satisfies the Kouchnirenko condition, then by Theorem 2.8 there exists $f:\left(\mathbf{C}^{n}, 0\right) \rightarrow(\mathbf{C}, 0)$, supp $f \subset M$, which has an isolated singularity at 0 . Hence $\Gamma_{+}(f) \subset \Gamma_{+}\left(\lambda_{M}\right)$ and by monotonicity of the Newton number (see for example [6]) we have $v\left(\lambda_{M}\right) \leq v(f)$. On the other hand, since $f$ has an isolated singularity at 0 , we have $v(f) \leq \mu(f)<\infty$ by [8, Théorème 1(i)]. Summing up $v\left(\lambda_{M}\right)$ is finite.

" $\Leftarrow$ " Now, suppose that $v\left(\lambda_{M}\right)<\infty$. Then by [8, Théorème 6.1] we may choose non-degenerate $f$ with supp $f=M$. Then $v(f)=v\left(\lambda_{M}\right)<\infty$. Therefore by Theorem 3.2 we get that $f$ has an isolated singularity at 0 . Hence, by Corollary 2.9 we get the assertion.

Remark 3.13. Gwoździewicz proved monotonicity of the Newton number for convenient function using [8, Théorème I (ii)] and semi-continuity of the Milnor number. It it is easy to generalize his result to the non-convenient case using only definition of the Newton number and simple properties of Newton diagram.

The following lemma has already been announced by Kouchnirenko [8, Subsection 6.5] (without proof).

Lemma 3.14. Let $S \subset \mathbf{R}^{n}$ be a d-dimensional simplex, $d \leq n-1$, and let $\operatorname{vert}(S)$ be the set of the vertices of $S$. Assume that $0 \notin$ aff $S$, the affine hull of $S$. Then every $f \in \mathbf{C}\left[z_{1}, \ldots, z_{n}\right]$ satisfying $\operatorname{supp}(f)=\operatorname{vert}(S)$ is non-degenerate.

Proof. First we consider the case $d=n-1$. Let $f=\sum_{v \in \operatorname{vert}(S)} a_{v} z^{v}$, where $a_{v} \neq 0$. The system of equations $\{\nabla f=0\}$ is equivalent to the system

$$
\left\{z_{1} \frac{\partial f}{\partial z_{1}}=\cdots=z_{n} \frac{\partial f}{\partial z_{n}}=0\right\}
$$

in $\left(\mathbf{C}^{*}\right)^{n}$. Since $z_{i} \frac{\partial f}{\partial z_{i}}=\sum_{v \in \operatorname{vert}(S)} a_{v} v_{i} z^{v}$, we see that this last system can be viewed as linear in unknowns $\left\{z^{v}\right\}$. This means that it has a non-zero solution in $\mathbf{C}^{n}$ if and only if $D:=\operatorname{det}\left[a_{v} v_{i}\right]_{\substack{v \in \operatorname{vert}(S) \\ 1 \leq i \leq n}}$ is zero. We have

$$
D=\prod_{v} a_{v} \cdot \operatorname{det}\left[v_{i}\right] .
$$

The assumption $0 \notin$ aff $S$ implies $\operatorname{dim}(\operatorname{span} S)=n$ so that the set $\operatorname{vert}(S)$ consists of $n$ linearly independent vectors. Hence, $\operatorname{det}\left[v_{i}\right] \neq 0$ and also $D \neq 0$ as the $a_{v}$ are non-zero. This means that the system $\{\nabla f=0\}$ has no solutions in $\left(\mathbf{C}^{*}\right)^{n}$. Moreover, every choice of a face $\sigma$ of the simplex $S$ corresponds to deletion of 
some columns in the matrix $\left[a_{v} v_{i}\right]_{v \in \operatorname{vert}(S), 1 \leq i \leq n}$. Such truncated matrix still has maximal possible rank which implies that $f$ is non-degenerate on $\sigma$. Hence, $f$ is non-degenerate.

In the general case, one can extend the $d$ dimensional simplex $S$ to an $(n-1)$-dimensional one and similarly add some missing terms to the function $f$ and in this way return to the first case.

Example 3.15. The assumption that $\operatorname{supp} f=\operatorname{vert}(S)$ cannot be omitted in the above lemma. Indeed, take $f\left(z_{1}, z_{2}\right)=z_{1}^{2}+2 z_{1} z_{2}+z_{2}^{2}$. Observe that supp $f \neq \operatorname{vert}(S)$ and $f=\left(z_{1}+z_{2}\right)^{2}$ is degenerate.

Also the assumption that $S$ is a simplex cannot be omitted. Indeed, take

$$
f\left(z_{1}, z_{2}, z_{3}, z_{4}\right)=z_{1}^{2}-z_{2}^{2}+z_{1} z_{3}^{2}+z_{2} z_{3}^{2} .
$$

We may observe that $S$ spanned by supp $f$ is not a simplex and supp $f=$ $\operatorname{vert}(S)$. Take $\phi(t)=\left(-t^{2} / 2, t^{2} / 2, t\right)$. Then $(\nabla f) \circ \phi=0$, so $f$ is degenerate.

From Lemma 3.14 and Theorem 3.1 we immediately get the following.

COROllary 3.16. Let $f:\left(\mathbf{C}^{n}, 0\right) \rightarrow(\mathbf{C}, 0)$ be a holomorphic function which has a singularity at 0 . Assume that all the faces $S \in \Gamma(f)$ are simplices and $\operatorname{supp} f_{S}=\operatorname{vert}(\sigma)$, the set of vertices of $S$. If supp $f$ satisfies the Kouchnirenko condition, then $f$ has an isolated singularity at 0 .

Example 3.17. Let $f\left(z_{1}, z_{2}, z_{3}\right)=z_{1}^{6} z_{3}+z_{2}^{4}+z_{3}^{12} z_{2}+z_{1} z_{3}^{2}+z_{2} z_{3}^{4}$. It is easy to check that all the faces $S \in \Gamma(f)$ are simplices and $\operatorname{supp} f_{S}=\operatorname{vert}(S)$. Moreover, it is easy to verify that supp $f$ satisfies the Kouchnirenko condition. Hence by the corollary above we infer that $f$ has an isolated singularity at 0 . Observe that $f$ is not convenient.

\section{Appendix}

Now, we find the constant $C$ in Lemma 3.6. Namely, we prove that $C \leq$ $\mathfrak{f}_{0}(f)+2$ (Proposition 4.2). First we give some definitions and theorems.

Let $F=\left(f_{1}, \ldots, f_{n}\right):\left(\mathbf{C}^{n}, 0\right) \rightarrow\left(\mathbf{C}^{n}, 0\right)$ be a holomorphic mapping having an isolated zero at the origin. We define the number

$$
l_{0}(F):=\inf \left\{\alpha \in \mathbf{R}_{+}: \exists C>0 \exists r>0 \forall\|z\|<r\|F(z)\| \geq C\|z\|^{\alpha}\right\}
$$

Let $f:\left(\mathbf{C}^{n}, 0\right) \rightarrow(\mathbf{C}, 0)$ be a holomorphic function which has an isolated singularity at 0 . We define the number $f_{0}(f)=l_{0}(\nabla f)$ and we call it the Łojasiewicz exponent $f$.

First we recall the following.

Lemma 4.1 ([17, Lemma 1.4]). Let $F, G:\left(\mathbf{C}^{n}, 0\right) \rightarrow\left(\mathbf{C}^{n}, 0\right)$ be holomorphic mappings in some neighbourhood of $0 \in \mathbf{C}^{n}$. Suppose that $F$ has an isolated zero. 
If $\operatorname{ord}(G-F)>l_{0}(F)$, then $G$ has an isolated zero and

$$
l_{0}(G)=l_{0}(F), \quad i_{0}(G)=i_{0}(F),
$$

where $i_{0}(F)$ denotes the multiplicity $F$ at $0 \in \mathbf{C}^{n}$.

Now, we give a constructive version of Lemma 3.6.

Proposition 4.2. Let $f:\left(\mathbf{C}^{n}, 0\right) \rightarrow(\mathbf{C}, 0), n \geq 2$ be a holomorphic function which has an isolated singularity at $0 . \quad$ Let $I \subset\{1,2, \ldots, n\}$ be a non-empty set such that $\Gamma_{+}(f) \cap O X_{i}=\emptyset$ for $i \in I$ and $\Gamma_{+}(f) \cap O X_{i} \neq \emptyset$ for $i \notin I$. Let $m_{i} \geq$ $\left[£_{0}(f)\right]+2, i \in I$. Then

$$
\begin{aligned}
v\left(f+\sum_{i \in I} z_{i}^{m_{i}}\right) & =v(f) \\
\mu\left(f+\sum_{i \in I} z_{i}^{m_{i}}\right) & =\mu(f) \\
£_{0}\left(f+\sum_{i \in I} z_{i}^{m_{i}}\right) & =£_{0}(f)
\end{aligned}
$$

Proof. Let $m_{i} \geq\left[£_{0}(f)\right]+2, i \in I$ and $f_{k}=f+\sum_{i \in I} z_{i}^{m_{i}}$. We begin with the proof of (8) and (9). We get

$$
\operatorname{ord}\left(\nabla f_{k}-\nabla f\right) \geq\left[£_{0}(f)\right]+1>£_{0}(f) .
$$

Hence by Lemma 4.1 we have $£_{0}\left(f_{k}\right)=£_{0}(f)$ and $\mu\left(f_{k}\right)=\mu(f)$.

Now we pass to the proof of (7). Since the Kouchnirenko non-degeneracy is a Zariski open condition (see for example [13, Appendix]), we may choose nondegenerate $\hat{f}$ with supp $\hat{f}=\operatorname{supp} f$. Since $f$ has an isolated singularity at 0 , by Corollary 2.9 we get that supp $f$ satisfies the Kouchnirenko condition. Hence supp $\hat{f}$ also satisfies the Kouchnirenko condition. Therefore by Theorem 3.1 we get that $\hat{f}$ has an isolated singularity at 0 . Since the Kouchnirenko nondegeneracy is a Zariski open condition we choose generic $\alpha_{i} \neq 0$ such that $\hat{f}_{k}=$ $\hat{f}+\sum_{i \in I} \alpha_{i} z_{i}^{m_{i}}$ is non-degenerate. We have

$$
\operatorname{ord}\left(\nabla \hat{f}_{k}-\nabla \hat{f}\right) \geq\left[£_{0}(\hat{f})\right]+1>£_{0}(\hat{f}) .
$$

Hence by Lemma 4.1 we have $\mu\left(\hat{f}_{k}\right)=\mu(\hat{f})$. Summing up by Corollary 3.10 , we get

$$
v\left(f+\sum_{i \in I} z_{i}^{m_{i}}\right)=v\left(\hat{f}_{k}\right)=\mu\left(\hat{f}_{k}\right)=\mu(\hat{f})=v(\hat{f})=v(f) .
$$

Example 4.3. The following example shows that in some cases the bound $\left[£_{0}(f)\right]+2$ for $m_{i}$ of Proposition 4.2 is the least possible. Indeed, take 
$f\left(z_{1}, z_{2}\right)=z_{2}^{6}+z_{1}^{3} z_{2}^{3}+z_{1}^{6} z_{2}$. One may check that $f$ is non-degenerate and $f$ has an isolated singularity at 0 . Using the main result of [11] we calculate $£_{0}(f)=6.5$ and by Corollary 3.10 we get $\mu(f)=v(f)=28$. Hence $\left[£_{0}(f)\right]+2=8$. Now take $\hat{f}=f+z_{1}^{7}$. Using the same techniques one may calculate $\mathfrak{f}_{0}(\hat{f})=6, \mu(\hat{f})=v(\hat{f})=27$.

Example 4.4. The following example shows that in some cases the bound $\left[\mathfrak{f}_{0}(f)\right]+2$ for $m_{i}$ of Proposition 4.2 is not the least possible. Indeed, take $f\left(z_{1}, z_{2}\right)=z_{1}^{8}+z_{1}^{2} z_{2}^{2}+z_{1} z_{2}^{3}$. One may check that $f$ is non-degenerate and $f$ has an isolated singularity at 0 . Using the main result of [11] we calculate $£_{0}(f)=7$ and by Corollary 3.10 we get $\mu(f)=v(f)=13$. Hence $\left[£_{0}(f)\right]+2=9$. Now take $f_{N}=f+z_{2}^{N}, \quad N \geq 5$. Using the same techniques one may calculate $£_{0}\left(f_{N}\right)=7, \mu\left(f_{N}\right)=v\left(f_{N}\right)=13$ for every $N \geq 5$.

Remark 4.5. For non-degenerate functions Fukui [5] gave the inequality

$$
£_{0}(f) \leq m_{0}(f)-1,
$$

where $m_{0}(f)$ is a combinatorial number calculated from $\Gamma_{+}(f)$. Also Abderrahmane [1] gave a similar estimation in terms of $v(f)$. In the case $n=2,3$ there are more exact formula for the Lojasiewicz exponent of non-degenerate holomorphic functions (see [11] and [14]).

Remark 4.6. One may also give an estimation of the constant $C$ in Lemmas 3.7 and 3.8 in terms of $m_{0}(f)$. Kouchnirenko [8, Théorème 3.7] also gave an estimation of the constant $C$ in generic version of Lemma 3.8, but his estimation is in general too large.

Acknowledgements. We would like to thank the anonymous referee for valuable comments.

\section{REFERENCES}

[ 1 ] O. M. Abderrahmane, On the Łojasiewicz exponent and Newton polyhedron, Kodai Math. J. 28 (2005), 106-110.

[2] V. I. Arnold, S. M. Gusein-Zade And A. N. Varchenko, Singularities of differentiable maps 1, Monographs Math. 82, Birkhäuser, Boston, 1985.

[ 3 ] S. Brzostowski, T. Krasiński and G. Oleksik, A conjecture on the Łojasiewicz exponent, J. Singul. 6 (2012), 124-130.

[4] J. Damon AND T. Gaffney, Topological trivality of deformations of functions and Newton filtrations, Invent. Math. 72 (1983), 335-358.

[ 5 ] T. FukuI, Łojasiewicz type inequalities and Newton diagrams, Proc. Amer. Math. Soc. 112 (1991), 1169-1183.

[6] J. Gwoździewicz, Note on the Newton number, Univ. Iag. Acta Math. 46 (2008), 31-33.

[7] C. Hertling and R. Kurbel, On the classification of quasihomogeneous singularities, J. Singul. 4 (2012), 131-153. 
[ 8 ] A. G. Kouchnirenko, Polyèdres de Newton et nombres de Milnor, Invent. Math. 32 (1976), $1-31$ (in French).

[9] A. G. Kouchnirenko, Criteria for the existence of a non-degenerate quasihomogeneous function with given weights, Usp. Mat Nauk 32 (1977), 169-170 (In Russian.).

[10] M. Kreuzer AND H. Skarke, On the classification of quasihomogeneous function, Commun. Math. Phys. 150 (1992), 137-147.

[11] A. LenarciK, On the Lojasiewicz exponent of the gradient holomorphic function, Banach Center Publications 44 (1998), 149-166.

[12] A. NÉmETHI, Invariants of Newton non-degenerate surface singularities, Compositio Math. 143 (2007), 1003-1036.

[13] М. OKA, On the bifurcation of the multiplicity and topology of the Newton boundary, J. Math. Soc. Japan 31 (1979), 435-450.

[14] G. Oleksik, The Lojasiewicz exponent of nondegenerate surface singularity, Acta. Math. Hungar. 138 (2013), 179-199.

[15] G. Oleksik, On combinatorial criteria for isolated singularities, Analytic and algebraic geometry, University of Lodz, 2013, 81-94.

[16] P. ORLIK AND R. RANDELl, The classification and monodromy of weighted homogeneous singularities, preprint, 1976 or 1977.

[17] A. Ploski, Sur l'exposant d'une application analytique II, Bull. Pol. Acad. Sci. Math. 33 (1985), 123-127 (in French).

[18] T. RodaK And S. Spodzieja, Effective formulas for the local Łojasiewicz exponent, Math. Z. 268 (2011), 37-44.

[19] K. SAIto, Quasihomogene isolierte Singularitäten von Hyperflächen, Invent. Math. 14 (1971), 123-142 (in German).

[20] K. Saito, Regular systems of weights and their associated singularities, Advanced studies in pure math. 8, Kinokuniya \& North Holland, 1987, 479-526.

[21] P. O. Shcherbak, O. P. Conditions for the existence of a non-degenerate mapping with a given support, Func. Anal. Appl 13 (1979), 154-155.

[22] C. T. C. WALL, Weighted homogeneous complete intersection, Progr. math. 134, Birkhäuser, Basel, 1996, 277-300.

[23] C. T. C. WALL, Newton polytopes and non-degeneracy, J. Reine Angew. Math. 509 (1999), $1-19$.

Szymon Brzostowski

FACUlty of Mathematics AND Computer SCIENCE

UNIVERSITY OF LODZ

BANACHA 22, 90-238 LoDZ

POLAND

E-mail: brzosts@math.uni.lodz.pl

Grzegorz Oleksik

FACUlty of Mathematics AND COMPUTER SCIENCE

UNIVERSITY OF LODZ

BANACHA 22, 90-238 LODZ

POLAND

E-mail: oleksig@math.uni.lodz.pl 